\title{
Doenças sexualmente transmissíveis e a vulnerabilidade da população do Alto Solimões, Amazonas, Brasil
}

\section{Sexually transmitted diseases and the vulnerability of the population in the Alto Solimões region, in Amazonas, Brazil}

\author{
leda Guedes Simões Coulibaly \\ Faculdade de Ciências Médicas da Santa Casa de São Paulo. São \\ Paulo, SP, Brasil. \\ E-mail: ieda9ı22@gmail.com

\section{José Cassio de Morais} \\ Faculdade de Ciências Médicas da Santa Casa de São Paulo. \\ Departamento de Saúde Coletiva. São Paulo, SP, Brasil. \\ E-mail: jcassiomळuol.com.br
}

\section{Regina Maria Giffoni Marsiglia}

Faculdade de Ciências Médicas da Santa Casa de São Paulo. Departamento de Saúde Coletiva. São Paulo, SP, Brasil.

E-mail: regina.marsiglia®fcmsantacasa.edu.br

\section{Patrícia Martins Montanari}

Faculdade de Ciências Médicas da Santa Casa de São Paulo. Departamento de Saúde Coletiva. São Paulo, SP, Brasil.

E-mail: patimontanariळgmail.com

\section{Correspondência}

leda Guedes Simões Coulibaly

Rua Dr. Urbano Pereira, 184, Vila Santa Teresa.

São Paulo, SP, Brasil. CEP 03566-020.

\section{Resumo}

Pessoas que apresentam sintomas de doenças sexualmente transmissíveis na região do Alto Solimões (Amazonas, Brasil), podem procurar farmácias mais próximas de onde residem para tratamento. Este artigo tem por finalidade mostrar como são tratadas e orientadas as pessoas que procuram pelo atendimento em farmácias em vez buscar os serviços de saúde, que são de mais difícil acesso na região. Para tal, realizou-se uma pesquisa de caráter qualitativo e emprego de uma amostra de conveniência. Na pesquisa de campo realizada em três dos nove municípios da região, utilizou-se o "Método do Cliente Misterioso" validado pelo protocolo Situational Analysis of Sexual Health in India (Sashi), desenvolvido pelo London School of Hygiene and Tropical Medicine, já utilizado na Índia, África e no Alto Solimões, seguido de entrevistas semiestruturadas com os mesmos participantes da etapa do cliente misterioso. Observou-se que, quando do uso do Método do Cliente Misterioso, os entrevistados indicaram e venderam antibióticos sem receita médica aos que os procuraram e, quando do uso das entrevistas semiestruturadas, essa prática não foi observada. Em ambas as abordagens, não houve orientação aos clientes sobre os riscos das doenças sexualmente transmissíveis para eles e seus parceiros sexuais. Concluímos com esse estudo que as pessoas que vivem no Alto Solimões estão vulneráveis a essas doenças, sendo necessário que se repense a questão da assistência médica e farmacêutica no âmbito das doenças sexualmente transmissíveis em regiões de difícil acesso do país.

Palavras-chave: Doenças Sexualmente Transmissíveis; Alto Solimões; Assistência Farmacêutica; Cliente Misterioso. 
People who present symptoms of sexually transmitted diseases in the Alto Solimões region (Amazonas, Brazil) can seek treatment at the pharmacies nearest to their homes. This article has the purpose of showing how people who seek assistance in pharmacies, instead of in the health care services, which are less accessible in the region, are treated and oriented. For this, we made a research of qualitative nature and used a convenience sample. Field research was performed in three of the nine municipalities of the region. The "Mystery Client Method" validated by the Situational Analysis of Sexual Health in India (Sashi) Protocol developed by the London School of Hygiene and Tropical Medicine, already used in India, Africa, and in the Alto Solimões region, was used, followed by semistructured interviews with the same participants of the mystery client phase. It was observed that, when using the Mystery Client Method, the respondents indicated and sold antibiotics without doctor's prescriptions to those who sought it, and when using the semi-structured interviews, this practice was not found. In both approaches, there was no orientation to the clients on the risks of the STDs for themselves and their sexual partners. With this study, we conclude that people living in the Alto Solimões region are vulnerable to such diseases, and it is necessary to rethink the issue of the medical and pharmaceutical assistance in the context of the sexually transmitted diseases in regions of difficult access in the country.

Keywords: Sexually Transmitted Diseases; Alto Solimões; Pharmaceutical Assistance; Mystery Client.

\section{Introdução}

A busca pela assistência farmacêutica para sanar problemas de saúde da população, em geral, é uma prática comum no Brasil e mais ainda em locais distantes de centros urbanos. Nesse artigo mostramos como são atendidas pessoas na região do Alto Solimões (Amazonas) que apresentam sintomas de doenças sexualmente transmissíveis (DSTs) e se reportam às farmácias em vez de irem ao médico, por falta de acesso a serviços de saúde ou mesmo por questões culturais.

A farmácia é um estabelecimento de prestação de serviços farmacêuticos de interesse público e/ou privado, destinado a prestar assistência farmacêutica e orientação sanitária individual ou coletiva, onde são processadas a manipulação e dispensa de produtos e correlatos com finalidade profilática, curativa, paliativa, estética, ou para fins de diagnóstico (CFF, 2001). Já a Assistência Farmacêutica é definida como grupo de atividades relacionadas com o medicamento, destinadas a apoiar as ações de saúde demandadas por uma comunidade envolvendo o abastecimento, o controle de qualidade, a segurança e a eficácia terapêutica dos medicamentos (Brasil, 2007).

No Brasil, os farmacêuticos são considerados como elos entre os medicamentos e a população, estão inseridos no sistema oficial de saúde e são procurados por serem considerados substitutos populares dos médicos, visto que são profissionais que podem ser acessados muito mais facilmente pela população, já que drogarias e farmácias são encontradas na maioria dos municípios mais distantes do país. Em muitas situações, o profissional que atende nos balcões das farmácias, tem sido o primeiro ponto de contato de pessoas com sintomas de doenças sexualmente transmissíveis (Garcia et al., 2012).

Há uma estimativa de que o número de pessoas acometidas por DSTs curáveis no mundo seja de 340 milhões ao ano (WHO, 2001). No Brasil, a gonorreia, a sífilis e a clamídia são consideradas as DSTs mais comuns, com cerca de 1.541.80o, 937.00o e 1.967.200 de casos, respectivamente ${ }^{1}$. Mas apenas a infecção 
pelo vírus da imunodeficiência humana (HIV), Aids, as hepatites virais, a infecção pelo HIV em gestantes e em crianças expostas ao risco vertical, sífilis adquirida, em gestantes e congênita, constituem doenças sexualmente transmissíveis de notificação compulsória. Para as demais patologias, não há necessidade de notificação, o que torna ainda mais difícil saber o que acontece em regiões remotas do país quando falamos da assistência às pessoas com DSTs.

Esse artigo é baseado nos achados da tese de doutorado desenvolvida por Coulibaly (2016) no Alto Solimões (Amazonas), região da tríplice fronteira entre Brasil, Colômbia e Peru. O foco do estudo foi conhecer como trabalhadores nos balcões de farmácias (balconistas ou farmacêuticos) orientam clientes que os procuram relatando que, após ter mantido relações sexuais com uma trabalhadora do sexo, apresentam sintomas de uretrite, patologia caracterizada por disúria, corrimento purulento e eritema uretral.

\section{Metodologia}

Trata-se de um estudo qualitativo, realizado em três municípios do Alto Solimões, que utilizou o Método do Cliente Misterioso e um roteiro com entrevistas semiestruturadas. A utilização desse método baseou-se no protocolo desenvolvido por Lambert et al. (2001) na London School of Hygiene and Tropical Medicine, intitulado protocolo Sashi (Situational Analysis of Sexual Health in India). O protocolo descreve como realizar diagnósticos situacionais que tomam as doenças sexualmente transmissíveis como eixo temático para a problematização da política de controle de agravos e, assim, realizar posterior intervenção. Partes do protocolo Sashi já haviam sido utilizadas em estudos na Índia, África e no Brasil, e publicados entre os anos de 2001 e 2012 (Lambert et al., 2001; Leiva et al., 2001; Souza; Silva Neto; Benzaken, 2012).

O Método do Cliente Misterioso é conhecido desde o início do século XX, tendo surgido nos Estados Unidos. Uma pessoa é orientada a simular ser um cliente que se utiliza dos serviços oferecidos pela empresa pesquisada e assim observar e anotar posteriormente como foi atendido. Tem sido bastante utilizado nos dias de hoje em empresas americanas e no Brasil, com o objetivo de avaliar serviços prestados por funcionários durante o atendimento a clientes, além das condições ambientais das empresas (Almeida et al., 2013).

$\mathrm{Na}$ área da saúde existem estudos realizados utilizando a metodologia para verificar a eficácia da assistência farmacêutica. Observa-se, através desse tipo de estudo, a possibilidade da venda de medicamentos sem prescrição médica, além das orientações dadas aos clientes por farmacêuticos e atendentes de farmácias (Volpato et al., 2005; Naves et al., 2008; Vacca; Niño; Reveiz, 2011; Xu; Almeida Neto; Moles, 2012; Chong; Aslani; Chen, 2014).

O protocolo Sashi, em seu item D, detalha o Método do Cliente Misterioso sugerindo que "o entrevistador" cliente misterioso deverá ser homem adulto, natural de região com costumes semelhantes aos do local de estudo, e que se faça entender durante a sua atuação que tem uma companheira mulher que poderá também estar doente. $\mathrm{O}$ item $\mathrm{D}$ do protocolo também detalha como ele deve agir, o que falar e como se comportar durante o atendimento.

No entanto, para esse estudo incluiu-se uma adaptação que tornou a análise dos resultados mais eficaz: a inclusão de um padrão ideal de respostas, esse padrão ideal é sugerido por empresas da área de serviços que tem utilizado o método para avaliar suas empresas e funcionários.

O padrão ideal de respostas desenvolvido para esse estudo teve como base o fato de que as pessoas que trabalham nas farmácias não podem vender antibióticos sem prescrição médica, devendo orientar o cliente para que se dirija a um serviço de saúde junto com seu parceiro sexual, para verificar a possibilidade de estar com alguma doença sexualmente transmissível (Anvisa, 2010, 2011; CFF, 2001). Assim, o padrão de respostas considerado ideal foi: não medicar; orientar o cliente para que procurasse um médico; orientar o cliente para que usasse preservativo nas suas relações sexuais; orientar acerca dos riscos de contrair o vírus HIV; orientar acerca da importância de realizar um teste para a detecção do HIV; orientar a ida da parceira ao serviço de saúde. Utilizou-se o mesmo padrão de respostas considerado ideal tanto para o Método Cliente Misterioso quanto para o roteiro de entrevistas. 
Em Manaus foi selecionado um homem nascido na região Amazônica, mas não no Alto Solimões, com idade de 45 de anos. Foi treinado na região do Alto Solimões para atuar como "entrevistador cliente misterioso". Esse entrevistador visitou as farmácias indicadas, relatando estar sentindo sintomas de uretrite que apareceram depois de manter relações sexuais com uma trabalhadora do sexo, comentando, ainda, que sua esposa estava preocupada com seu estado de saúde.

O estudo foi realizado através de amostra por conveniência em farmácias de três municípios do Alto Solimões localizadas em pontos de grande fluxo de pessoas. Nesses locais, os autores Souza, Silva Neto e Benzaken (2012) localizaram e mapearam bares, hotéis, motéis e quartos que podem ser alugados por hora, sugerindo pontos de prostituição próximos aos locais de lazer e diversão dos municípios.

Para esse estudo, que foi realizado nos meses de maio e abril de 2015, foram selecionadas doze farmácias nesses mesmos pontos mapeados, porém a pesquisa foi realizada somente em onze estabelecimentos, já que um deles estava com suas dependências alagadas, uma vez que o período em que estivemos na região era de chuvas intensas. Dentre esses estabelecimentos, conseguiu-se aplicar a Metodologia do Cliente Misterioso e, posteriormente, entrevistas com roteiro prévio, em onze funcionários que atendiam os clientes nos balcões dessas farmácias.

\section{Entrevistas}

Após a atuação do "entrevistador cliente misterioso" em abril de 2015, outro pesquisador foi até os mesmos estabelecimentos farmacêuticos visitados em maio de 2015 e realizou entrevistas com os atendentes de farmácia que estavam disponíveis naquele momento (não necessariamente o mesmo atendente que foi entrevistado pelo cliente misterioso), com algumas perguntas abertas e outras fechadas:

Se um cliente com os sintomas abaixo viesse pedir ajuda, qual o conselho que você daria?

"Sou casado e mantive relações sexuais sem uso de preservativo faz duas semanas com uma trabalhadora do sexo. Também mantive relações sexuais com minha esposa há uma semana e não usei preservativo. Há três dias, notei uma secreção branca, grossa na região uretral e não tomei qualquer medicação. Tive uma doença sexualmente transmissível há cerca de um ano, mas não sei dizer o nome da doença".

As demais perguntas, todas com respostas sim e não, foram: as pessoas pedem conselhos sobre como tratar os sintomas das doenças sexualmente transmissíveis? Quantas pessoas trabalham na farmácia? Aqui trabalha um farmacêutico? Aqui vende preservativos? - Estão em locais visíveis e disponíveis? Você costuma sugerir que o seu cliente use preservativo?

\section{Questões éticas}

A pesquisa foi aprovada pelo Comitê de Ética e Pesquisa da Irmandade da Santa Casa de Misericórdia de São Paulo, CAEE 37746114.4.0ooo.5479 em 28 de janeiro de 2015. Este estudo foi dispensado de solicitar a assinatura do Termo de Consentimento Livre e Esclarecido (TCLE) durante a atuação do cliente misterioso; porém, durante as entrevistas, todos os participantes assinaram o TCLE. Todos os estabelecimentos farmacêuticos e seus funcionários tiveram seus dados e sua localização preservados e não serão divulgados. Apesar de sabermos quais os municípios participaram e, através da amostra por conveniência, qual região da cidade foi selecionada, não foram divulgadas quais farmácias dessas regiões participaram.

\section{o local do estudo: Alto Solimões, Amazonas}

O Alto Solimões é uma importante microrregião do estado do Amazonas e está situada no extremo sudoeste do estado. Possui $214.217 \mathrm{~km}^{2}$ sendo composta pelos municípios de Amaturá, Atalaia do Norte, Benjamin Constant, Fonte Boa, Jutaí, Santo Antônio do Içá, São Paulo de Olivença, Tabatinga e Tonantins (Silva Neto, 2010).

Os municípios de Tabatinga, Benjamin Constant e Atalaia do Norte fazem parte da tríplice fronteira com Colômbia e Peru, sendo que o município de Tabatinga é o mais populoso da região. É cidade 
gêmea com a capital do Amazonas Colombiano, a cidade de Letícia, com a qual forma uma conurbação, ou seja, um único espaço urbano ligado por uma via, a avenida da amizade. Há um intenso comércio entre os dois países com grande número de pessoas atravessando de um lado ao outro; pessoas que são naturais da região ou que vêm de outros países, como os Haitianos, por exemplo, que após o terremoto de 2010 atravessaram a fronteira para o Brasil em grande quantidade. A passagem intensa de pessoas através dessa fronteira traz uma grande preocupação com o "leva e traz" de agentes patogênicos e acabam tornando o local um pronto estratégico para pesquisas em saúde.

Figura I - Mapa do Estado do Amazonas e Alto Solimões em destaque em branco, Brasil, 2016

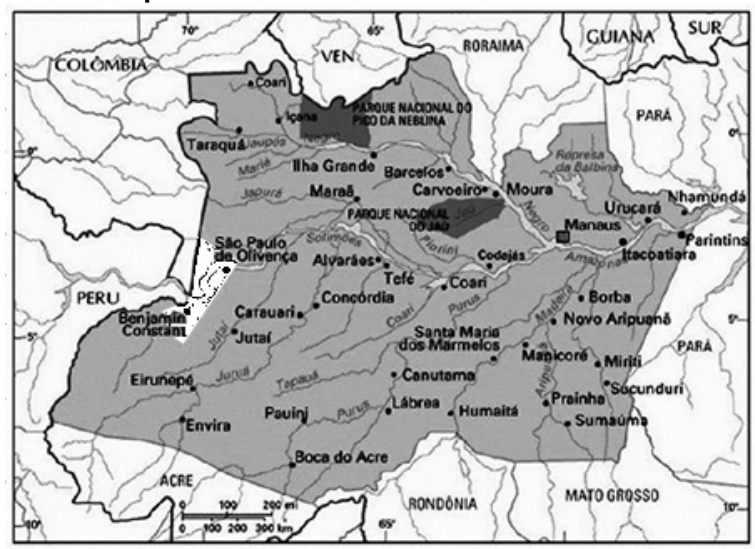

A região também apresenta um grande contingente de povos indígenas nas zonas urbana e rural. Segundo dados do IBGE², em 2010, havia dez municípios no Amazonas com a maior concentração dessa população no país, desses, seis estão no Alto Solimões. Além dos povos indígenas, vivem na região agricultores, pescadores, migrantes das regiões norte e nordeste, militares, missionários e madeireiros (Peiter, 2005).

Observamos, ainda, que os municípios do Alto Solimões estão entre os mais vulneráveis do estado do Amazonas em relação à pobreza. Atalaia do Norte e Santo Antônio do Içá, por exemplo, apresentam Índice de Desenvolvimento Humano Municipal (IDHM) muito baixo, e apenas Tabatinga se apresenta com o índice médio3.

A maioria dos municípios tem densidade demográfica abaixo de 3 habitantes $/ \mathrm{Km}^{2}$, excetuando-se os municípios de Benjamin Constant e Tabatinga, com densidade de 3,80 e 16,21 habitantes $/ \mathrm{Km}^{2}$ respectivamente. A região possui dois aeroportos, um no município de Tabatinga e outro no município de São Paulo de Olivença. As distâncias entre os municípios não são tão grandes quando comparamos com locais onde há estradas; Tabatinga e Benjamin Constant, por exemplo, estão distantes $19 \mathrm{~km}$, porém, a viagem é realizada com um barco chamado "voadeira", mas, de Tabatinga para os outros municípios mais distantes, o percurso deve ser feito em barcos maiores com custos também maiores.

Essas embarcações saem de Manaus, vão para Tabatinga e depois seguem rumo aos outros municípios. De Tabatinga para Fonte Boa, por exemplo, cuja distância é de $460 \mathrm{~km}$, a viagem de barco dura um dia e treze horas, e o barco sai do porto de Tabatinga a cada 21 dias. Se o munícipe decide viajar de lancha, a saída da embarcação é semanal e a viagem dura treze horas, no entanto, o preço da passagem também é mais alto.

Em se tratando da atenção à saúde da população, a região do Alto Solimões apresenta-se com doze unidades de atenção à saúde indígena, 71 centros de saúde, treze postos de saúde, dez unidades hospitalares mistas, sessenta equipes de saúde da família e 103 médicos registrados no Conselho Nacional de Saúde. No entanto, apesar desses dados nos remeterem à ideia de um quantitativo de profissionais e estabelecimentos de saúde adequados para os cerca de 240.000 habitantes, as características da região com sua elevada extensão territorial, deslocamentos difíceis pela escassez de transporte e alagamentos, e os indicadores sociais ruins, sugerem que a população possa ter dificuldade em acessar os serviços

2 IBGE - INSTITUTO BRASILEIRO DE GEOGRAFIA E ESTATÍSTICA. Informações sobre a distribuição da população autodeclarada indígena no território brasileiro com base nos resultados censitários IBGE. Brasília, DF, 201o. Disponível em: <http://indigenas.ibge. gov.br/>. Acesso em: 6 abr. 2015.

3 ADHB - ATLAS DO DESENVOLVIMENTO HUMANO NO BRASIL, 2010. Disponível em: <http://bit.ly/2iu7U6R>. Acesso em: 16 jan. 2017. 
médicos e por isso buscariam ajuda nas farmácias. Ao buscar as farmácias, existe o questionamento sobre como essas pessoas são orientadas acerca da possibilidade de estar com uma DST.

\section{Resultados}

Após visitar as onze das doze farmácias selecionadas entre os meses de abril e maio de 2015, procedeu-se a análise das respostas do cliente misterioso e das entrevistas, optando pela técnica de análise de conteúdo (Bogdan; Biklen, 1994). Sendo assim, a análise do material coletado foi dividida em: análise do conteúdo das respostas registradas pelo entrevistador que fez papel de cliente misterioso e algumas anotações que fez em seu diário de campo. Em seguida, foi realizada a análise do conteúdo das respostas das entrevistas com roteiro e, ao final, elaborado um quadro sintético para comparação entre as respostas obtidas através dos dois instrumentos de entrevista aplicados aos atendentes nas farmácias visitadas.

Durante sua atuação nas farmácias, o cliente misterioso pôde observar que dentre os onze aten- dentes das farmácias visitadas, nenhum mencionou a possibilidade de ele estar com uma doença sexualmente transmissível, dois orientaram a procurar ajuda médica, dez indicaram antibióticos e o venderam sem receita médica com valores que variavam entre R\$ 8, oo e R\$ 43,oo. Em relação ao uso de preservativos, orientações sobre os riscos de contrair HIV e sobre o tratamento da parceira, não houve nenhuma orientação (Quadro 1).

Durante a etapa das entrevistas com roteiro, os onze entrevistados responderam que encaminhariam o cliente ao médico, cinco declararam já ter atendido pacientes com aqueles sintomas; três enfatizaram que não vendem antibióticos sem receita médica, mesmo essa questão não estando entre as perguntas (Quadro 1).

Em média, as farmácias visitadas têm três funcionários, todas vendem preservativos que se encontram em local visível, próximo ao caixa ou no balcão. Somente em duas farmácias os entrevistados disseram que oferecem preservativos aos clientes. Em todas as farmácias há farmacêuticos no quadro de funcionários, porém, durante a entrevista esse profissional não estava presente em nenhum dos estabelecimentos.

\begin{tabular}{|c|c|c|}
\hline Padrão considerado ideal & $\begin{array}{l}\text { Padrão verificado- } \\
\text { cliente misterioso }\end{array}$ & $\begin{array}{l}\text { Padrão verificado- } \\
\text { entrevistas }\end{array}$ \\
\hline Não medicar & $\begin{array}{l}\text { Dez indicaram antibióticos } \\
\text { Dez venderam antibióticos }\end{array}$ & $\begin{array}{l}\text { Onze funcionários das farmácias } \\
\text { disseram que não medicariam }\end{array}$ \\
\hline Orientar a procura por um médico & $\begin{array}{l}\text { Dois funcionários das farmácias orientaram } \\
\text { que fosse ao médico. } \\
\text { Uma delas apresentou padrão misto - vendeu } \\
\text { antibiótico e sugeriu a ida ao médico }\end{array}$ & $\begin{array}{l}\text { Onze funcionários das farmácias } \\
\text { disseram que o encaminhariam ao } \\
\text { médico }\end{array}$ \\
\hline Orientar o uso de preservativo & $\begin{array}{l}\text { Nenhuma orientou; porém uma perguntou se } \\
\text { ele usava preservativo }\end{array}$ & $\begin{array}{l}\text { Todas vendem preservativo, mas não } \\
\text { costumam oferecer }\end{array}$ \\
\hline $\begin{array}{l}\text { Orientar sobre os riscos de contrair } \\
\text { HIV }\end{array}$ & Nenhuma orientou & Nenhuma orientou \\
\hline $\begin{array}{l}\text { Orientar sobre a realização de } \\
\text { exames para detecção do HIV }\end{array}$ & Nenhuma orientou & Nenhuma orientou \\
\hline Orientar o tratamento da parceira & Nenhuma orientou & Nenhuma orientou \\
\hline
\end{tabular}




\section{Discussão}

Um dos grandes desafios da humanidade é controlar, reduzir os efeitos ou eliminar sofrimentos causados por enfermidades (Brasil, 2007). Esse estudo nos mostra uma realidade muitas vezes escondida em municípios e populações que vivem distantes dos grandes e médios centros urbanos do país: pobreza, dificuldade de acesso a serviços médicos por questões diversas e a busca pela solução dos problemas de saúde de acordo com os padrões culturais e experiências locais para o enfretamento e cura das enfermidades.

$\mathrm{O}$ isolamento vivido por muitas pessoas nos interiores do país pode contribuir para a busca de soluções alternativas na tentativa de minimizar problemas de saúde. Os moradores de lugares como o descrito nesse estudo podem decidir por ir à farmácia ou buscar os agentes da medicina popular como primeira opção para tratamento de suas doenças: são recursos de localização mais próxima de sua moradia, onde o atendimento é imediato sem a necessidade de cadastro ou filas de espera. Além disso, muitas vezes esses profissionais são conhecidos da população, por estarem sempre presentes na cidade, morar junto à comunidade ou conhecer particularmente os moradores no entorno do seu estabelecimento.

Nesse estudo, o entrevistador que atuou como cliente misterioso relata em seu diário de campo a sua percepção sobre a ida à farmácia como primeira opção das pessoas que vivem na região, afirmando:

Se eu estivesse doente de verdade, eu também iria na farmácia (Cliente Misterioso).

Por outro lado, deve-se levar em conta que a Anvisa (Agência Nacional de Vigilância Sanitária), através das RDC 44/2010 e RDC 20/2011, dispõe sobre o controle de medicamentos à base de substâncias classificadas como antimicrobianos: a prescrição dos medicamentos deve ser realizada por profis- sionais legalmente habilitados, ou seja, os clientes das farmácias só podem comprar antibióticos se a receita for prescrita por profissional médico (Anvisa; 2010, 2011).

Neste estudo, observa-se que os atendentes das farmácias pesquisadas venderam o antibiótico sem a devida prescrição médica ao entrevistador cliente misterioso. Contudo, não podemos apenas considerar a ilegalidade da ação, pois o profissional na farmácia mostra que tem conhecimento de que o cliente terá dificuldades em acessar o serviço de saúde, não só pelas distâncias, mas também pela deficiência do sistema de saúde do país.

A moça me disse para ir ao médico, só que ela falou para ir no particular, senão ia demorar muito (Cliente Misterioso).

Sabe-se que não é fácil conseguir atendimento médico imediato em muitos lugares no Brasil. Vale registrar que, apesar da quantidade de médicos e serviços de saúde nos parecer adequados para a região, ao fazermos uma busca nos jornais do Alto Solimões utilizando os termos "falta de médicos, reclamações sobre serviços de saúde e falta de medicamentos", termos estes repetidos para cada um dos nove municípios, encontrou-se entre os anos de 2012 e 2015, em três jornais online locais, nove reportagens cujos conteúdos tratam de problemas como falta de médicos, falta de medicamentos e cobranças as ações governamentais para melhoria da saúde na região (Coulibaly, 2016).

Em relação ao custo dos medicamentos, dentre as farmácias que venderam antibióticos, quatro cobraram o valor mais alto (R\$ 43,oo). Lembrando que, segundo dados do IBGE 4 , em todos os municípios da região, mais de $25 \%$ da população vive em domicílios vulneráveis à pobreza. Essa situação nos aponta um impasse: de um lado o paciente vulnerável às doenças sexualmente transmissíveis e, do outro, um sistema de saúde que exige a apresentação de receita médica para obtenção do antibiótico, mas que não garante que o paciente a conseguirá gratui-

4 IBGE - INSTITUTO BRASILEIRO DE GEOGRAFIA E ESTATÍSTICA. Informações sobre a distribuição da população autodeclarada indígena no território brasileiro com base nos resultados censitários IBGE. Brasília, DF, 201o. Disponível em: <http://indigenas.ibge. gov.br/>. Acesso em: 6 abr. 2015 . 
tamente e facilmente em serviços de saúde próximos de onde mora, e nem que conseguirá o medicamento a um custo razoável, se houver a necessidade de pagar por ele.

A venda de antibióticos sem receita médica não é exclusividade do Alto Solimões. Estudos realizados em Brasília (Naves et al., 2008) nos mostra o mesmo padrão encontrado no Alto Solimões. Além disso, em países como Arábia Saudita (Abdulhak et al., 2011), Indonésia (Puspitasari; Faturrohmah; Hermansyah, 2011) e Colômbia (Vacca; Niño; Reveiz, 2011), observou-se que, diante de um possível portador de DSTs, a prática mais comum dos profissionais que atuam nas farmácias dessas regiões foi receitar antibióticos aos pacientes em vez de encaminhá-los ao médico.

Esse fato nos remete a outro problema que vem sendo alvo de preocupações no mundo todo: a resistência microbiana aos antibióticos. Problema grave que está em pauta nas políticas públicas de uso racional de medicamentos, pois as taxas mais elevadas de resistência bacteriana parecem surgir nos países onde se consomem antibióticos de forma abusiva. Lembrando que neste estudo o entrevistador que atuou como cliente misterioso entrou em onze farmácias e, em dez, conseguiu o medicamento sem receita médica. Trata-se de uma facilidade que põe em risco a saúde de toda coletividade.

Outra questão importante é a não orientação sobre os riscos da doença e da necessidade de tratamento do parceiro sexual. Em ambos os instrumentos de coleta de dados, o parceiro sexual, nesse caso, a companheira do cliente misterioso, foi ignorada pelos atendentes em todas as farmácias, o que nos leva a considerar o quanto as mulheres da região estão vulneráveis às DSTs.

Diante do cenário apresentado, a implementação de ações educativas voltadas aos trabalhadores das farmácias visando minimizar a falta de orientação aos clientes e a prevenção e controle das DSTs, talvez seja uma opção para iniciar a abordagem de uma parte do problema, ou seja, a falta de orientações.

Quando falamos de farmácias e ações educativas, um estudo realizado em Campo Grande (Mato Grosso do Sul) revelou que, após intervenção educativa nas farmácias, as orientações acerca das DSTs melhoraram de qualidade, porém, a venda dos medicamentos sem receita permaneceu inalterada (Ajalla; Castro, 2003). Resultado semelhante ocorreu em estudo realizado em Brasília e Taguatinga (Naves et al., 2008), pois, após a intervenção educativa junto às farmácias, as orientações para que o usuário procurasse o tratamento do parceiro sexual e informações sobre o uso de preservativo não surtiu efeito, além de que a venda de medicamentos sem receita médica continuou inalterada. Por outro lado, é preciso considerar que, para resultarem mudanças positivas, as intervenções educativas precisam ser monitoradas e avaliadas periodicamente.

\section{Conclusão}

Através dos resultados deste estudo pudemos identificar cinco problemas no Alto Solimões: venda de antibióticos em farmácias sem receita prescrita por um profissional médico; alto custo dos medicamentos oferecidos à população que apresenta índices alarmantes de pobreza; risco de disseminação de doenças sexualmente transmissíveis por falta de diagnóstico e orientações sobre uso de preservativo; o risco da proliferação de bactérias resistentes pelo uso irracional de antimicrobianos e negligência em relação à saúde sexual da mulher.

Os profissionais e atendentes que trabalham nas farmácias estão inseridos no sistema oficial de saúde e são muitas vezes procurados em primeiro lugar quando a população se sente doente. Por esse motivo, precisam se proteger de possíveis penalidades advindas das agências reguladoras. Essa é a melhor explicação sobre a negativa deles quando são questionados sobre a venda ilegal do antibiótic durante a entrevista: o medo de penalidades.

A questão da venda de antibióticos com a obrigatoriedade da apresentação da receita médica precisa ser repensada, pois não há garantia de que essa receita será conseguida pelo usuário e também não há garantia de que o medicamento realmente só será vendido com a apresentação da receita.

Não se pode tratar pessoas em lugares tão desiguais no país como iguais; é necessário adequar a assistência médica e repensar a assistência farmacêutica no âmbito das doenças sexualmente transmissíveis para o Alto Solimões. Ao mesmo tempo, 
é imprescindível o desenvolvimento de campanhas educativas na região, tanto para a população quanto para os profissionais nas farmácias, pois, para que haja efetividade no controle das DSTs, é necessário abrir os olhos para as particularidades das regiões remotas do país.

\section{Referências}

ABDULHAK, A. A. B. et al. Non prescribed sale of antibiotics in Riyadh, Saudi Arabia: a cross sectional study. BMC Public Health, London, v. 11, n. 1, p. 538, 2011.

AJALLA, M. E. A.; CASTRO, L. C. Contribuição para melhoria da prática farmacêutica no atendimento de pacientes com queixas referentes a doenças sexualmente transmissíveis, em Campo Grande-MS. Revista de Ciências Farmacêuticas, Araraquara, v. 24, n. 2, p. 121-130, 2003.

ALMEIDA, R. S. et al. A influência da padronização e do controle sobre o funcionário do setor de serviços. In: Encontro Anpad. 37, 2013, Rio de Janeiro. Anais... Rio de Janeiro: Associação Nacional de Pós-Graduação e Pesquisa em Administração, 2013. Disponível em: <http://bit. ly/2iuv7Wv> Acesso em: 6 abr. 2015.

ANVISA - AGÊNCIA NACIONAL DE VIGILÂNCIA SANITÁRIA. Resolução RDC $N^{o} 44$, de 26 de outubro de 2010. Dispõe sobre o controle de medicamentos à base de substâncias classificadas como antimicrobianos, de uso sob prescrição médica, isoladas ou em associação e dá outras providências. Brasília, DF, 2010. Disponível em: <http://bit.ly/2jh7ON5>. Acesso em: 8 abr. 2015.

ANVISA - AGÊNCIA NACIONAL DE VIGILÂNCIA SANITÁRIA. Resolução RDC $n^{\circ} 20$, de 5 de maio de 2011. Dispõe sobre o controle de medicamentos à base de substâncias classificadas como antimicrobianos, de uso sob prescrição, isoladas ou em associação. Brasília, DF, 2011. Disponível em: <http://bit.ly/2iEggaB >. Acesso em: 8 abr. 2015 .

BOGDAN, R.; BIKLEN, S. Investigação qualitativa em educação: uma introdução à teoria e aos métodos. Porto: Porto, 1994.
BRASIL. Conselho Nacional de Secretários de Saúde. Assistência farmacêutica no SUS. Brasília, DF, 2007. Disponível em: <http://bit.ly/2j11lEU〉.

Acesso em: 16 jan. 2017.

CHONG, W. W.; ASLANI, P.; CHEN, T. F. Pharmacist-patient communication on use of antidepressants: a simulated patient study in community pharmacy. Research in Social \& Administrative Pharmacy, Amsterdam, v. 10, n. 2, p. 419-37, 2014.

CFF - CONSELHO FEDERAL DE FARMÁCIA. Resolução ${ }^{\circ}$ 357, de 20 de abril de 2001. Aprova o regulamento técnico de Boas Práticas de Farmácia. Diário Oficial da União, Brasília, DF, 27 abr. 2001. Seção 1, p. 24-3o. Disponível em: <http:// bit.ly/2jıbdıy> Acesso em: 6 nov. 2015.

COULIBALY, I. G. S. Atendentes de farmáciase agentes da medicina popular na região do Alto Solimões frente aos pacientes com queixas de doenças sexualmente transmissiveis. 2016. Tese (Doutorado em Ciências da Saúde) -Faculdade de Ciências Médicas da Santa Casa de São Paulo, São Paulo, 2016.

GARCÍA, P. J. et al. Improved STD Syndrome management by a network of clinicians and pharmacy workers in Peru: The PREVEN Network. Plos One, San Francisco, v. 7, n. 10, p. e4775o, 2012.

LAMBERT, H. et al. Situational Analysis of Sexual Health in India (SASHI): implementation package. Technical Report. LSHTM Research Online, London, 2001. Disponível em: <http://bit. ly/2jXvDNk>. Acesso em: 7 mar. 2015.

LEIVA, A. et al. Management of sexually transmitted diseases in urban pharmacies in The Gambia. International Journal of STD \& AIDS, London, v. 12, n. 7, p. 444-452, 2001.

NAVES, J. A. S. et al. Práticas de atendimento a DST nas farmácias do Distrito Federal, Brasil: um estudo de intervenção. Cadernos de Saúde Pública, Rio de Janeiro, v. 24, n. 3, p. 577-586, 2008.

PEITER, P. C. A geografia da saúde na faixa de fronteira continental do Brasil na passagem do milênio. 2005. Tese (Doutorado em Geografia) Universidade Federal do Rio de Janeiro, Rio de Janeiro, 2005. 
PUSPITASARI, H. P.; FATURROHMAH, A.;

HERMANSYAH, A. Do Indonesian community pharmacy workers respond to antibiotics requests appropriately? Tropical Medicine \& International Health, Oxford, v. 16, n. 7, p. 840-846, 2011.

SILVA NETO, A. L. Caracterização geográfica, epidemiológica e da organização dos serviços de saúde da tríplice fronteira Brasil/Colômbia/ Peru. 2010. Tese (Doutorado em Saúde Pública) Centro de Pesquisas Aggeu Magalhães, Fundação Oswaldo Cruz, Recife, 2010.

SOUZA. I. S.; SILVA NETO, A. L.; BENZAKEN, A. S. Uso de mapas temáticos e promoção de saúde sexual do Alto Solimões - Amazonas - Brasil. Hygeia, Uberlândia, v. 8, n. 14, p. 157-167, 2012.

VACCA, C. P.; NIÑO, C.Y.; REVEIZ, L. Restricción de la venta de antibióticos en farmacias de
Bogotá, Colômbia: estudio descriptivo. Revista Panamericana de Salud Publica, Washington, DC, v. 3o, n. 6, p. 586-591, 2011.

VOLPATO, D. E. et al. Use of antibiotics without medical prescription. Brazilian Journal of Infeccion Disease, Salvador, v. 9, n. 4, p. 288-291, 2005.

WHO - WORLD HEALTH ORGANIZATION. Global prevalence and incidence of selected curable sexually transmitted infections: overview and estimates. Geneva: WHO, 2001. Disponível em: <http://bit.ly/1i5BCCw>. Acesso em: 14 abr. 2015.

XU, T.; ALMEIDA NETO, A. C. ; MOLES, R. J. A systematic review of simulated-patient methods used in community pharmacy to assess the provision of non-prescription medicine. International Journal of Pharmacy Practice, New Jersey, v. 20, n. 5, p. 307-319, 2012.

\section{Contribuição dos autores}

Coulibaly concebeu o projeto de pesquisa, trabalhou na coleta de dados, na interpretação dos resultados e na redação final do artigo. De Moraes, foi o responsável pela orientação do trabalho, trabalhou na interpretação dos resultados, na redação e na revisão final do artigo. Marsiglia trabalhou na interpretação dos dados e na revisão final do artigo. Montanari trabalhou na interpretação dos dados e na revisão final do artigo.

Recebido: 24/04/2016

Reapresentado: $11 / 10 / 2016$

Aprovado: 26/10/2016 